\title{
Implante percutâneo de valva aórtica: mito ou realidade?
}

\author{
Percutaneous aortic aortic valve replacement: myth or reality?
}

\author{
Eduardo Keller SAADI ${ }^{1}$
}

\section{Resumo}

A substituição valvar por prótese metálica ou biológica com o auxílio de circulação extracorpórea é o procedimento padrão-ouro para o tratamento da estenose aórtica calcificada. Embora os resultados sejam excelentes com a cirurgia convencional, alguns pacientes com idade avançada, doenças associadas, reoperações e disfunção ventricular esquerda grave apresentam alto risco cirúrgico. Nos últimos anos, técnicas de tratamento percutâneo foram desenvolvidas. A presente revisão tem por objetivo analisar a literatura desde o desenvolvimento experimental até a aplicação clínica desta nova modalidade de tratamento para pacientes com estenose aórtica grave e alto risco cirúrgico. $O$ implante percutâneo de valva aórtica hoje vem sendo realizado por alguns centros e o cirurgião cardiovascular envolvido no tratamento das doenças valvares deve fazer parte deste desenvolvimento.

Descritores: Estenose da valva aórtica. Valva aórtica. Contenedores.

\begin{abstract}
Aortic valve replacement with mechanical or biological prosthesis with extracorporeal circulation is the goldstandard for the treatment of calcific aortic stenosis. Although the results are excellent with the conventional approach some elderly patients, with multiple high-risk comorbid conditions, reoperations and severe left ventricular dysfunction have high surgical risk. During the last years percutaneous techniques have been developed. The present study aim to analyse the literature, since the experimental development untill clinical application of this novel treatment in patients with high surgical risk aortic stenosis. Percutaneous implantation of aortic valve prosthesis is beeing done in some centers and the cardiovascular surgeon that treats valve disease should be involved in this development.
\end{abstract}

Descriptors: Aortic valve stenosis. Aortic valve. Stents.

1. Doutorado; Professor Adjunto de Cirurgia Cardiovascular da Universidade Federal do Rio Grande do Sul (UFRGS)/Hospital de Clínicas de Porto Alegre (HCPA). Professor de Cirurgia Cardiovascular da Universidade Luterana do Brasil (ULBRA). Membro Titular da SBCCV.

Trabalho realizado na Universidade Luterana do Brasil (ULBRA), Porto Alegre, RS, Brasil. 


\section{INTRODUÇÃO}

A estenose aórtica calcificada ocorre entre $2 \%$ e $5 \%$ dos indivíduos idosos e é a principal causa de cirurgia para troca valvar nos Estados Unidos da América e Europa. A cirurgia cardíaca com o emprego de circulação extracorpórea e substituição valvar aórtica por prótese mecânica ou biológica é o procedimento de escolha nestes casos. A troca valvar aórtica com o auxílio da circulação extracorpórea é realizada com mortalidade muito baixa e pouca morbidade e só em algumas situações especiais acarreta alto risco cirúrgico. A cirurgia convencional propicia melhora sintomática e aumento da sobrevida na maioria dos pacientes, com baixo risco cirúrgico [1]. Este procedimento é o padrão-ouro para o tratamento da estenose e/ou insuficiência aórtica. Entretanto, em pacientes com doenças associadas, muito idosos, reoperações e com disfunção ventricular grave, a mortalidade pode chegar até $50 \%[2,3]$.

$\mathrm{Na}$ última década, surgiram tentativas com procedimentos menos invasivos, como a derivação do ápice do ventrículo esquerdo para a aorta e valvuloplastia aórtica com cateter-balão, para tentar tratar pacientes com risco cirúrgico alto [4-7].

O tratamento percutâneo das doenças valvares aórticas com implante de valvas por cateter com "stents" vem sendo testado como alternativa [8-13].

Após a utilização com relativo sucesso para tratar estenose aórtica neonatal, a dilatação com cateter-balão foi tentada em pacientes com estenose aórtica calcificada, com resultados decepcionantes, e foi praticamente abandonada.

A partir daí, o conceito da troca valvar não cirúrgica evoluiu. Inicialmente foi aplicada à valva pulmonar, porque o acesso é mais fácil e esta tolera mais facilmente resultados não tão perfeitos quanto a valva aórtica. Depois de um curto período de experimentação animal [9], os primeiros implantes de "stents" com valva pulmonar foram relatados por Bonhoeffer et al. [14], em 2000, em pacientes com disfunção de conduto do ventrículo direito para a artéria pulmonar. Atualmente, mais de 100 pacientes foram tratados pelos autores mencionados com apenas uma morte relacionada ao procedimento e bons resultados neste grupo pediátrico [15,16]. Cribier et al. [17], em 2002, realizaram o primeiro implante em humanos de uma prótese valvar aórtica expansível por balão, em paciente com estenose aórtica grave considerado inoperável pelas co-morbidades associadas. Desde então, relatos com esta nova técnica têm mostrado que este procedimento é factível [18-22].

Nos primeiros procedimentos, as valvas montadas em cateter com "stent" eram liberadas no anel aórtico através da abordagem transseptal anterógrada, mas tecnicamente este procedimento era difícil, principalmente para posicionar adequadamente a valva no anel aórtico, tendo sido abandonado.

O acesso retrógrado via artéria femoral parece mais lógico, mas uma limitação importante tem sido aquela relacionada à condição do acesso arterial. Inicialmente bainhas de 24 French (F) ou maiores eram necessárias para liberar as próteses. Atualmente, na terceira geração destes dispositivos, já existem introdutores com diâmetro de 18 F. O acesso vascular periférico nestes pacientes idosos com oclusão aterosclerótica aorto-ilíaca ou vasos ilíaco-femorais finos é um dos problemas a ser superado. Outros problemas relacionados com o método são: o risco de embolização cálcica durante a pré-dilatação da valva aórtica, comprometimento dos óstios das artérias coronárias, migração do "stent” valvado, "leak" para-valvar, hemólise, baixo débito cardíaco no momento da dilatação e da liberação e a pouca durabilidade da prótese.

É difícil para todo o cirurgião que tenha experiência com pacientes que apresentam estenose aórtica calcificada imaginar que é possível pré dilatar estas valvas pétreas. Mas, contrariamente à lógica cirúrgica, nos últimos três anos, alguns trabalhos experimentais foram publicados e a experiência clínica inicial demonstra que este procedimento é possível, sendo recentemente estendido à valva mitral [23,24].

Uma alternativa ao acesso arterial retrógrado tem sido a liberação anterógrada, com cateter, transapical pelo ventrículo esquerdo através de minitoracotomia, com o coração batendo. O grupo da Universidade de British Columbia, em Vancouver, no Canadá, publicou experiência clínica com ótimos resultados usando esta técnica [25].

Naturalmente, devido à rápida expansão e desenvolvimento da área, muita controvérsia foi criada. Com o objetivo de tentar estabelecer o real "estado da arte" e normatizar as indicações para implante valvar percutâneo a Society of Thoracic Surgeons (STS), a American Association for Thoracic Surgery (AATS), em conjunto com a Society for Cardiovascular Angiography and Interventions (SCAI) publicou uma posição oficial [26]. Este texto alerta para as precauções que devem ser tomadas para se evitar o uso indiscriminado destes procedimentos, ressaltando que muitas questões críticas permanecem sem resposta, incluindo a durabilidade dos dispositivos. Salientam que tecnologias experimentais não são justificadas em pacientes nos quais não existam diretrizes publicadas com indicação clara ou em situações de cirurgia profilática até que dados de segurança e efetividade sejam publicados baseados em ensaios clínicos bem desenhados.

Mesmo com as ressalvas feitas, o método tem sido utilizado em alguns centros, empregando protocolos clínicos experimentais, em pacientes sem condições de serem submetidos a cirurgia convencional, onde dois cirurgiões cardiovasculares assinam termo contra-indicando a cirurgia aberta.

Os resultados iniciais tem sido razoáveis e com o grande interesse e investimento das empresas na área, o rápido avanço tecnológico na fabricação das valvas e de cateteres deve facilitar o procedimento e expandir as indicações. A maior parte das valvas é composta de três folhetos de pericárdio bovino 
ou eqüino, montadas com “stent” balão expansível ou autoexpansíveis, com liga metálica de aço inoxidável ou nitinol. Atualmente, mais de 20 empresas trabalham no desenvolvimento destes substitutos valvares e a CribierEdwards ${ }^{\circledR}$, assim como a CoreValve ${ }^{\circledR}$, já vêm sendo utilizadas em séries clínicas regularmente, de modo percutâneo e transapical.

O sistema com prótese auto-expansível apresenta várias vantagens potenciais em relação aos dispositivos expansíveis por balão. Além de teoricamente estar associado a menor incidência de vazamento para-valvar, permite tratar também pacientes com insuficiência aórtica. Outra vantagem potencial seria a maior durabilidade da prótese, já que o trauma causado aos folhetos das valvas relacionado à dilatação com balão seria eliminado [18,20,21].

Em dezembro de 2005, Lamarche et al. [27] realizaram implante de valva aórtica percutânea em Montreal, no Canadá, em paciente portadora de estenose aórtica grave, disfunção ventricular e fibrose pulmonar, com contra-indicação para cirurgia convencional. Foi colocada uma prótese autoexpansível CoreValve (Paris, França), por via arterial retrógrada, com o auxílio de circulação extracorpórea fêmoro-femoral. A evolução da paciente foi boa e os autores chamam a atenção para a importância da abordagem multidisciplinar nestes casos. Em 2006, Grube et al. [28] relataram uma série de 25 pacientes submetidos a implante percutâneo de valva aórtica utilizando circulação extracorpórea percutânea fêmoro-femoral. Neste estudo, pacientes com estenose aórtica sintomáticos, com área valvar menor que $1 \mathrm{~cm}$, foram considerados para inclusão. O implante de valva CoreValve foi realizado sob anestesia geral e suporte com circulação extracorpórea fêmoro-femoral. O acompanhamento incluiu avaliação clínica e ecocardiográfica, 15 e 30 dias após o procedimento, para avaliação dos resultados a curto prazo. Vinte e cinco pacientes sintomáticos com várias co-morbidades (mediana de EuroScore, $11 \%$ ) foram selecionados. O sucesso no procedimento foi atingido em 21 (84\%) dos pacientes. O implante resultou em redução significativa do gradiente ( $p<0,0001)$. Eventos maiores cardiovasculares e neurológicos ocorreram em oito (32\%) pacientes, incluindo cinco (20\%) mortes. Entre os 18 pacientes que tiveram alta hospitalar, não ocorreram efeitos adversos nos primeiros 30 dias. Os autores concluem que o implante percutâneo da prótese valvar aórtica auto-expansível CoreValve em pacientes de alto risco é factível e, quando implantada com sucesso, resulta em redução importante do gradiente e melhora clínica a curto prazo.

Em 2007, foi publicada uma série de 50 pacientes tratados de modo percutâneo, com até um ano de acompanhamento, mostrando bons resultados neste período e maior sucesso terapêutico nos últimos 25 casos, demonstrando a importância da curva de aprendizagem e a rápida evolução dos dispositivos [29].

Atualmente, com base nos estudos acima referidos, podemos afirmar que o implante percutâneo de valva aórtica é um procedimento que vem sendo aplicado, porém ainda encontra-se em fase de avaliação. No Brasil, a Agência Nacional de Vigilância Sanitária (ANVISA) ainda não liberou nenhum destes produtos. Muito temos a evoluir, mas este é um procedimento que veio para ficar. O que se pode discutir são as indicações e qual o real espaço que estes procedimentos menos invasivos devem ocupar na terapêutica clínica das doenças valvares. Apesar do excelente resultado da cirurgia valvar convencional, o avanço da ciência é rápido. Estamos vivendo em tempos exponenciais. O cirurgião cardiovascular envolvido com o tratamento das doenças valvares deve estar aberto à inovação. Também não deve se deixar levar pelo entusiasmo exagerado e procurar sempre avaliar os resultados à luz de estudos clínicos a médio e longo prazos bem conduzidos. É evidente que nós devemos aplicar a ciência com rigor para manter a segurança dos pacientes. Por outro lado, esta é mais uma área da Medicina onde a colaboração estreita entre o cirurgião cardiovascular e o cardiologista intervencionista é fundamental para o bem do paciente.

\section{REFERÊNCIAS}

1. Rajamannan NM. Calcific aortic stenosis: a disease ready for prime time. Circulation. 2006;114(19):2007-9.

2. Roques F, Nashef SA, Michel P, Gauducheau E, de Vincentiis C, Baudet E, et al. Risk factors and outcome in European cardiac surgery: analysis of the EuroSCORE multinational database of 19030 patients. Eur J Cardiothorac Surg. 1999;15(6):816-23.

3. Culliford AT, Galloway AC, Colvin SB, Grossi EA, Baumann FG, Esposito R, et al. Aortic valve replacement for aortic stenosis in persons aged 80 years and over. Am J Cardiol. 1991;67(15):1256-60.

4. Cooley DA, Lopez RM, Absi TS. Apicoaortic conduit for left ventricular outflow tract obstruction: revisited. Ann Thorac Surg. 2000;69(5):1511-4.

5. Sweeney MS, Walker WE, Cooley DA, Reul GJ. Apicoaortic conduits for complex left ventricular outflow obstruction: 10year experience. Ann Thorac Surg. 1986;42(6):609-11.

6. Renzulli A, Gregorio R, De Feo M, Ismeno G, Covino FE, Cotrufo M. Long-term results of apico-aortic valved conduit for severe idiopathic hypertrophic subaortic stenosis. Tex Heart Inst J. 2000;27(1):24-8. 
7. Vassiliades TA Jr. Off-pump apicoaortic conduit insertion for high-risk patients with aortic stenosis. Eur J Cardiothorac Surg. 2003;23(2):156-8.

8. Andersen HR, Knudsen LL, Hasenkam JM. Transluminal implantation of artificial heart valves: description of a new expandable aortic valve and initial results with implantation by catheter technique in closed chest pigs. Eur Heart J. 1992;13(5):704-8.

9. Bonhoeffer P, Boudjemline Y, Saliba Z, Hausse AO, Aggoun Y, Bonnet D, et al. Transcatheter implantation of a bovine valve in pulmonary position: a lamb study. Circulation. 2000;102(7):813-6.

10. Boudjemline Y, Bonhoeffer P. Steps toward percutaneous aortic valve replacement. Circulation. 2002;105(6):775-8.

11. Boudjemline Y, Bonhoeffer P. Percutaneous implantation of a valve in the descending aorta in lambs. Eur Heart J. 2002;23(13):1045-9.

12. Sochman J, Peregrin JH, Pavcnik D, Timmermans H, Rosch J. Percutaneous transcatheter aortic disc valve prosthesis implantation: a feasibility study. Cardiovasc Intervent Radiol. 2000;23(5):384-8.

13. Cribier A, Eltchaninoff H, Borenstein N. Transcatheter implantation of balloon expandable prosthetic heart valves: early results in an animal model. Circulation. 2001;104:II-552.

14. Bonhoeffer P, Boudjemline Y, Saliba Z, Merckx J, Aggoun Y, Bonnet D, et al. Percutaneous replacement of pulmonary valve in a right-ventricle to pulmonary-artery prosthetic conduit with valve dysfunction. Lancet. 2000;356(9239):1403-5.

15. Khambadkone S, Coats L, Taylor A, Boudjemline Y, Derrick G, Tsang V, et al. Percutaneous pulmonary valve implantation in humans: results in 59 consecutive patients. Circulation. 2005;112(8):1189-97.

16. Attmann T, Quaden R, Jahnke T, Muller-Hulsbeck S, Boening A, Cremer J, et al. Percutaneous pulmonary valve replacement: 3-month evaluation of self-expanding valved stents. Ann Thorac Surg. 2006;82(2):708-13.

17. Cribier A, Eltchaninoff H, Bash A, Borenstein N, Tron C, Bauer F, et al. Percutaneous transcatheter implantation of an aortic valve prosthesis for calcific aortic stenosis: first human case description. Circulation. 2002;106(24):3006-8.

18. Cribier A, Eltchaninoff H, Tron C, Bauer F, Agatiello C, Sebagh L, et al. Early experience with percutaneous transcatheter implantation of heart valve prosthesis for the treatment of end-stage inoperable patients with calcific aortic stenosis. J Am Coll Cardiol. 2004;43(4):698-703.

19. Bauer F, Eltchaninoff H, Tron C, Lesault PF, Agatiello C, Nercolini D, et al. Acute improvement in global and regional left ventricular systolic function after percutaneous heart valve implantation in patients with symptomatic aortic stenosis. Circulation. 2004;110(11):1473-6.

20. Webb JG, Chandavimol M, Thompson CR, Ricci DR, Carere RG, Munt BI, et al. Percutaneous aortic valve implantation retrograde from the femoral artery. Circulation. 2006;113(6):842-50.

21. Cribier A, Eltchaninoff H, Tron C, Bauer F, Agatiello C, Nercolini D, et al. Treatment of calcific aortic stenosis with the percutaneous heart valve: mid-term follow-up from the initial feasibility studies: the French experience. J Am Coll Cardiol. 2006;47(6):1214-23.

22. Grube E, Laborde JC, Zickmann B, Gerckens U, Felderhoff T, Sauren B, et al. First report on a human percutaneous transluminal implantation of a self-expanding valve prosthesis for interventional treatment of aortic valve stenosis. Catheter Cardiovasc Interv. 2005;66(4):465-9.

23. Ma L, Tozzi P, Huber CH, Taub S, Gerelle G, von Segesser LK. Double-crowned valved stents for off-pump mitral valve replacement. Eur J Cardiothorac Surg. 2005;28(2):194-9.

24. Boudjemline Y, Pineau E, Borenstein N, Behr L, Bonhoeffer P. New insights in minimally invasive valve replacement: description of a cooperative approach for the off-pump replacement of mitral valves. Eur Heart J. 2005;26(19):2013-7.

25. Lichtenstein SV, Cheung A, Ye J, Thompson CR, Carere RG, Pasupati S, et al. Transapical transcatheter aortic valve implantation in humans: initial clinical experience. Circulation. 2006;114(6):591-6.

26. Vassiliades TA Jr., Block PC, Cohn LH, Adams DH, Borer JS, Feldman T, et al. The clinical development of percutaneous heart valve technology: a position statement of the Society of Thoracic Surgeons (STS), the American Association for Thoracic Surgery (AATS), and the Society for Cardiovascular Angiography and Interventions (SCAI). Ann Thorac Surg. 2005;79(5):1812-8.

27. Lamarche Y, Cartier R, Denault A, Basmadjian A, Berry C, Laborde JC, et al. Implantation of the CoreValve percutaneous aortic valve. Ann Thorac Surg. 2007;83(1):284-7.

28. Grube E, Laborde JC, Gerckens U, Felderhoff T, Sauren B, Buellesfeld L, et al. Percutaneous implantation of the CoreValve self-expanding valve prosthesis in high risk patients with aortic valve disease: the Siegburg first-in-man study. Circulation. 2006;114(15):1616-24.

29. Webb JG, Pasupati S, Humphries K, Thompson C, Altwegg L, Moss R, et al. Percutaneous transarterial aortic valve replacement in selected high-risk patients with aortic stenosis. Circulation. 2007;116(7):755-63. 